\title{
Accuracy of MLP Based Data Visualization Used in Oil Prices Forecasting Task
}

\author{
Aistis Raudys \\ Institute of Mathematics and Informatics \\ Akademijos 4, Vilnius 08633, Lithuania \\ aistis@raudys.com
}

\begin{abstract}
We investigate accuracy, neural network complexity and sample size problem in multilayer perceptron (MLP) based (neuro-linear) feature extraction. For feature extraction we use weighted sums calculated in hidden units of the MLP based classifier. Extracted features are utilized for data visualisation in 2D and 3D spaces and interactive formation of the pattern classes. We show analytically how complexity of feature extraction algorithm depends on the number of hidden units. Sample size - complexity relations investigated in this paper showed that reliability of the neuro-linear feature extraction could become extremely low if number of new features is too high. Visual interactive inspection of data projection may help an investigator to look differently at the forecasting problem of the financial time series.
\end{abstract}

Keywords: Feature extraction, Data mapping, Sample size, Neural network.

\section{Introduction}

Feature extraction, feature selection and data visualization are very important phases while solving pattern recognition (PR) and forecasting tasks. We need to reduce a number of features in order to make pattern recognition system simpler, cheaper both in a design phase and in production. Dimensionality reduction is also very important while diminishing the small sample problem: with a decrease in a number of the features the sample size / dimensionality ratio increases making the classifier design problem easier.

In numerous PR tasks, the pattern classes are not specified a priori. On a basis of the problem knowledge, the designer ought to specify pattern classes. An example is pattern classification of images in biomedical research where physicians often cannot specify the pattern classes, in other words, distinguish unhealthy tissues from healthy ones. Similar problems we meet in forecasting of economic time series. The Efficient Market Hypothesis [1] states that in efficient market, the prices reflect all the information available from the market. Thus, statistically significant forecast can be made only in situations where either the market is not efficient enough in terms of information processing or the forecasting method is unexpected for other participants. Possible success may be obtained in case of unexpected formulation of the forecasting problem. Original way of forming of the pattern classes could play very important 
role here. To solve this task the researcher needs to inspect statistical data and generate a number of hypothesis that support the statistical times series data at hand.

The dimensionality reduction methods can be categorized by evaluation function used to calculate the feature subset quality and a search method employed to minimize (maximize) selected performance measure. There is a vast amount of literature of feature extraction and selection, data mapping topics. It is useless to present a serious review in a short conference paper. An interested reader is referred to well known reviews, e.g. [2-4] and a recent taxonomy of data mapping methods presented in the author's paper [5].

We consider how data visualisation can be used in class formation task. We also investigate small sample size problem in the neuro-linear feature extraction, 2/3D data visualisation and neural network complexity. The hypothesis formation about possible split of the data is performed in interactive mode by visual analysis of data in $2 \mathrm{D}$ and 3D data mappings. A number of attempts with diverse data mapping methods should be carried out before a suitable solution is obtained.

\section{Neuro-linear Feature Extraction}

In this section we present formal definition of the feature extraction (FE) procedure where the complexity of FE algorithm directly depends on the number of hidden units. This FE method performs linear feature extraction with nonlinear performance criterion. This peculiarity allows to take a new look at the complexity of FE process. To find optimal data transformation weights, standard three layer MLP with additional regularization term is trained and the first hidden layer weights are used as coefficients in linear FE: $r$ new features, $y_{1}, y_{2}, \ldots, y_{r}$, are weighted sums, $y_{s}$, calculated in $r$ hidden neurons [5]:

$$
y_{s}=\boldsymbol{w}_{s}^{T} \boldsymbol{x}+w_{s 0} \quad s=1,2, \ldots, r
$$

where $\boldsymbol{w}_{s}^{T}=\left(w_{s 0}, w_{s 1}, w_{s 2}, \ldots, w_{s p}\right)$ are weights of $s$-th hidden layer neuron (index “"T, denotes transposition operation), $p$ is dimensionality of the input feature space, $\boldsymbol{x}$ is $p$-dimensional input vector.

We see that MLP can actually be interpreted as linear mapping method with nonlinear SLP in its output stage. The new feature space depends on complexity of decision boundary. In the case of the two hidden units - two new features $(r=2)$ are obtained. The maximum complexity of decision boundary in 2D space is determined by shape " $\_$". The complexity of DB may be higher if more than two features would be extracted. In Fig. 1a we have decision boundary (DB) of the MLP based classifier with 3 hidden neurons trained by the Levenberg-Marquardt method. It is more non-linear as "angle shaped" boundary " $\_$" in case of two hidden units (boundary 1 (in dashes) in Fig. 1b). Positive side of neuro-linear feature extraction is the possibility to control the complexity of the decision boundary. We can: a) change the number of hidden layers, b) utilize the weight decay regularization term, c) purposefully proportionally decrease of weights, $w_{s 0}, w_{s 1}, w_{s 2}, \ldots, w_{s p}$, of each single neuron. The latter technique allows change the smoothness of decision boundary and inspect unknown structure of the data better. We will see that potential to control complexity is very important having in mind that training sample size is fixed (usually "small"). 
The neuro-linear feature extraction is rather similar to popular Foley - Sammon (sometimes called linear discriminant analysis feature extraction) feature mapping algorithm [6,7] which belongs to the group of supervised methods (wrapper approach). Here the standard Fisher linear classifier (FLC) [2,3] is used to create a linear decision boundary which helps to extract the first new feature. A new feature is a distance of vector $\boldsymbol{x}$ to discriminant hyperplane, $\boldsymbol{w}_{\mathrm{FC}}^{T} \boldsymbol{x}+w_{\mathrm{FLC} 0}=0$, where $\boldsymbol{w}_{\mathrm{FLC}}$ and $w_{\mathrm{FLC} 0}$ are the weights of FLC. The feature extraction procedure is repeated on the rest of the orthogonal features until there are no features left. Thus, the first extracted feature is a direction where pattern classes are best separated linearly. The second feature is the direction where classes are separated slightly worse, and so on.
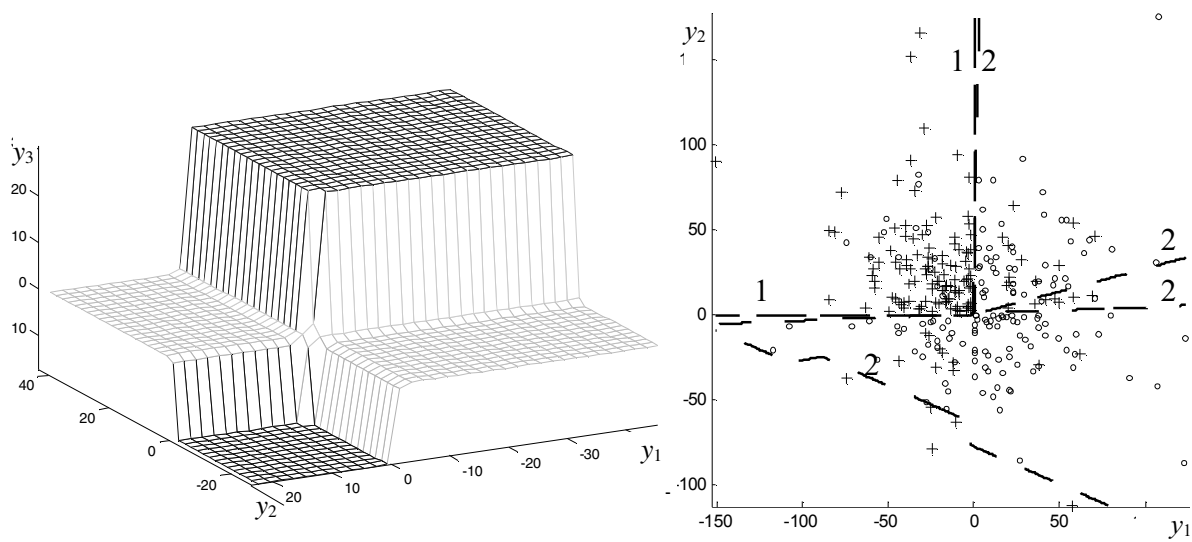

Fig. 1. Nonlinear decision boundaries of MLP: a) MLP with 3 hidden units in 3D new feature $y_{1}, y_{2}$ and $y_{3}$, space (resubstitution error estimate $\mathrm{P}_{\mathrm{R}}=0.12$, hold out error estimate $\mathrm{P}_{\mathrm{HO}}=0.34$ ). b) $1-\mathrm{DB}$ of MLP with 2 hidden units in new $2 \mathrm{D}$ space $\left(\mathrm{P}_{\mathrm{R}}=0.16, \mathrm{P}_{\mathrm{HO}}=0.33\right), 2-\mathrm{DB}$ MLP with 5 hidden units $\left(\mathrm{P}_{\mathrm{R}}=0.15, \mathrm{P}_{\mathrm{HO}}=0.36\right)$.

The Foley - Sammon mapping creates new features derived independently to other ones. This is disadvantage of Foley - Sammon method. Meanwhile, in neuro-linear feature extraction, minimization of performance criterion is performed simultaneously for all new features. In the Foley - Sammon mapping, optimization is performed sequentially: for the first new feature at first, then for the second one, etc.

Feature extraction often is utilized for data mapping into 2D or 3D spaces (data mapping) in order to perform preliminary data analysis and formation of pattern classes in interactive mode. In addition to wrapper approach, the filter approach based FE methods are used too. In most powerful of them, the projection pursuit method, one optimizes selected "indexes of interestingness" and looks for interesting lowdimensional projections of high-dimensional data.

\section{Small Sample Properties}

Traditionally in FE one utilizes the same data set for data mapping and for analysis of the data. If sample size is small and input dimensionality, $p$, is high, obviously we are 
examining inappropriate projection of the data in such a case. In Fig. 1b we have seen that distribution of training vectors in new feature space shows clear data structure: the data can be separated by " $\Gamma$ " shaped DB marked by 1 . Disappointingly, we could not observe any structure while inspecting test data vectors in the same $2 \mathrm{D}$ space.

Regrettably, an accuracy of feature extraction was not considered in the literature yet. Feature extraction is one of the most important steps in pattern recognition, therefore, small sample properties have to be studied here too. In present section, we will apply multivariate statistical analysis technique in order to evaluate accuracy of evaluation of seperability between distinct areas of the features space belonging to different pattern classes.

In MLP training, some of the hidden layer neurons can produce outputs either very close to 0 or to 1 (if sigmoid activation function, output $=1 /\left(1+\exp \left(-y_{s}\right)\right.$, is utilized in MLP training). Thus only a part of training vectors affects training process of separate hidden units. Just a subset of data influences training of each single neuron. In Fig. 1 we have seen that after training, decision boundary 1 in the new feature, $y_{1}, y_{2}, \ldots, y_{r}$, space is composed of intersecting linear parts (lines in 2D case, planes in 3D case). Thus, for a start let us assume that in neuro-linear feature extraction, weighted sums, $y_{s}=\boldsymbol{w}_{s}^{T} \boldsymbol{x}+w_{s 0},(s=1,2, \ldots, r)$ corresponding to each hidden unit are in fact standard Fisher classifiers formed in certain distinct portions of training data, $\Omega_{1}, \Omega_{2}, \ldots, \Omega_{r}$. Note that under some special unrestrictive conditions in single layer perceptron (SLP) training, we obtain standard Fisher linear classifier [4, 8]. Consequently the weights of new linearly extracted features corresponding to $s$-th portion $\Omega_{s}$ of training data can be expressed as

$$
\boldsymbol{w}_{s}=\mathbf{S}_{s}^{-1}\left(\overline{\boldsymbol{x}}_{s}^{(1)}-\overline{\boldsymbol{x}}_{s}^{(2)}\right), w_{s 0}=-1 / 2 \boldsymbol{w}_{s}^{T}\left(\overline{\boldsymbol{x}}_{s}^{(1)}+\overline{\boldsymbol{x}}_{s}^{(2)}\right),
$$

where $\overline{\boldsymbol{x}}_{s}^{(1)}, \overline{\boldsymbol{x}}_{s}^{(2)}$ and $\mathbf{S}_{s}$ are estimates of mean vectors and a pooled covariance matrix.

Let weighted sum (1) with weights (2) is used as new feature. Let us consider training set based estimates of distances $\hat{\delta}_{s}^{1}$ and $\hat{\delta}_{s}^{2}$ of the first and second class mean vectors in portion $\Omega_{s}$, from discriminant boundary, $\boldsymbol{w}_{s}^{T} \boldsymbol{x}+w_{s 0},=0$, normalized by standard deviation of $\boldsymbol{w}_{s}^{T} \boldsymbol{x}+w_{s 0}$. Rather simple, however, tedious and lengthy multivariate algebra (we omit derivations in this paper) results that $\hat{\delta}_{s}^{1}$ and $\hat{\delta}_{s}^{2}$ may be expressed as

$$
\hat{\delta}_{s}^{1}=\frac{\delta_{s}^{1} \sqrt{\left(1+\frac{p}{\delta_{s}^{2}} \frac{N_{1 s}+N_{2 s}}{N_{1 s} N_{2 s}}\right) \frac{N_{2 s}+N_{1 s}}{N_{2 s}+N_{1 s}-p}}}{\sqrt{1+\frac{p}{\delta_{s}^{2}} \frac{N_{1 s}-N_{2 s}}{N_{1 s} N_{2 s}}}}, \quad \delta_{s}^{2}=\frac{\delta_{s}^{2} \sqrt{\left(1+\frac{p}{\delta_{s}^{2}} \frac{N_{1 s}+N_{2 s}}{N_{1 s} N_{2 s}}\right) \frac{N_{2 s}+N_{1 s}}{N_{2 s}+N_{1 s}-p}}}{\sqrt{1+\frac{p}{\delta_{s}^{2}} \frac{N_{2 s}-N_{1 s}}{N_{1 s} N_{2 s}}}},
$$

where $\delta_{s}^{1}$ and $\delta_{s}^{2}$ are true normalized distances, $N_{1 s}$ and $N_{2 s}$ are numbers of training vectors of the first and second class in data portion $\Omega_{s}$.

Derivation of Equations (3) was based on crude assumption that the $\Omega_{s}$-th portion of training vectors belongs to two multivariate Gaussian distributions sharing common covariance matrix. Moreover, we ignored terms of order $p N_{1 S}^{-2}, p N_{2 s}^{-2}$. 
Numerical calculation according to Eq. (3) indicates that in finite sample case, training sample based distances $\hat{\delta}_{s}^{1}$ and $\hat{\delta}_{s}^{2}$ seem larger as hypothetical true distances, $\delta_{s}^{1}$ and $\delta_{s}^{2}$. It means that the data could seem well separable even if in reality it is inseparable at all.

Especially dangerous is a situation when $N_{1 s}$ and $N_{2 s}$ are close to $p$, the input dimensionality, $\delta_{s}^{1}$ and/or $\delta_{s}^{2}$, being very small. Let $\delta_{s}^{1}=0.01$ (no separation between classes), input dimensionality $p=50$ and $N_{2}=N_{1}=145$. Then $\hat{\delta}_{s}^{1}=0.913$. If only a half of training vectors affect hyperplane $\boldsymbol{w}_{s}^{T} \boldsymbol{x}+w_{s 0}=0\left(N_{2 s} \approx N_{1 s} \approx 72\right), \hat{\boldsymbol{\delta}}_{s}^{1}=1.45$. If we have three new features and assume that only $1 / 4^{\text {th }}$ part of the data affect hyperplane $\left(N_{2 s}=N_{1 s}=36\right), \hat{\delta}_{s}^{1}=2.98$. Actual distance was $\delta_{s}^{1}=0.01$. It means that from $2 \mathrm{D}$ mapping we conclude erroneously: "very good separation!".

Equations (3) indicate also that the bias of training sample based distances, $\hat{\delta}_{s}^{1}$ or $\hat{\delta}_{s}^{2}$, may increase in portion of training data $\Omega_{s}$, where $N_{2 s}>>N_{1 s}\left(\right.$ or $\left.N_{2 s}<<N_{1 s}\right)$. Such situation is difficult to avoid in practice since there are no arguments to expect that in each subset, $\Omega_{s}$, samples sizes $N_{2 s} \approx N_{1 s}$. Thus, in order to have a truthful picture of the data's structure, in each portion of the data, we need to have $N_{1 s}>>p$ and $N_{2 s}>>p$.

One more alternative is to add a noise to training vectors while extraction features by means of MLP training. Numerous simulation studies have shown that in order to minimize distortion of training data a $k$-nearest neighbour (colored) noise injection [9] is a useful tool. In this approach, to each training vector, say $\boldsymbol{x}_{z}$, one finds closest $k$ neighbours in training set of the same pattern class. In this subspace one adds a noise and increases a number of training vectors artificially. Then one uses the new expanded data set to train MLP for subsequent feature extraction. More details can be found in $[4,9]$. It is a way of introducing informal information which asserts that a space between neighbouring training vectors of one pattern class in not empty. In Fig. 2 we have an illustration obtained by means of utilization of a noise injection technique for FE. We see the same data structure both in training and test sets. For other identical conditions the test set error was reduced from $\mathrm{P}_{\mathrm{HO}}=0.36$ without noise injection (see Fig. 1b) to 0.26 . So, a noise injection approach helped us not to give up to temptation to utilize untruthful structure of the data's presented in Fig. 1b.

\section{Forecasting of Huge Changes in the Oil Prices}

Our studies indicated that neuro-linear linear feature extraction is very powerful tool to extract features and obtain erroneous conclusions if sample size - complexity considerations are ignored. Theory and experiment show that additional utilization of pseudo-validation set obtained by a noise injection technique can be useful in validation of the data analysis results.

In this paper the FE technique was already illustrated by commenting the classification of two pattern classes obtained from time series data describing changes in the oil prices: (a) West Texas Int. Cushing, (b) Natural Gas-Henry Hub., (c) Fuel 
Oil, No.2 (NY), (d) Gasoline, Unld. Reg. Non-Oxy (NY), and (e) American Stock Exchange (AMEX) oil price index during 2923 days in a period Nov 1, 1993 - Jan 12,2005 . Forecasting of the oil prices is very important economic and almost political task which has objectives both to gain in financial bargains and assisting in creating computerized models of financial and political World development [10-12].
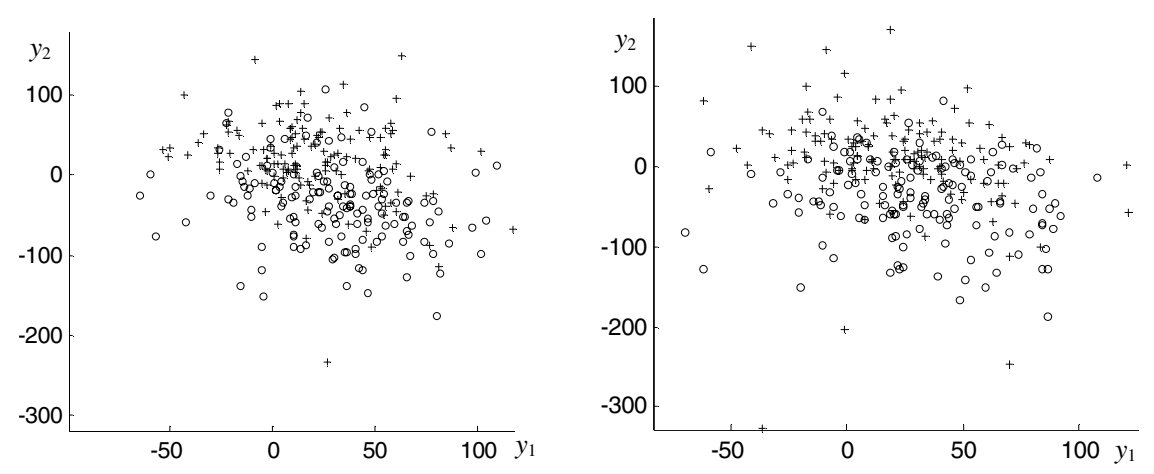

Fig. 2. Distribution of training (left) and test (right) vectors in $2 \mathrm{D}$ new feature, $y_{1}, y_{2}$, space obtained after training MLP with two hidden units with pseudo artificial training set created by noise injection procedure with: $\sigma_{\mathrm{n}}=0.5, n i_{n n}=5$ and $k=2\left(\mathrm{P}_{\mathrm{R}}=0.23, \mathrm{P}_{\mathrm{HO}}=0.28\right.$ after training MLP in 50D input feature space; $\mathrm{P}_{\mathrm{R}}=0.20, \mathrm{P}_{\mathrm{HO}}=0.26$ after additional training in $2 \mathrm{D}$ space.

The changes in the oil prices were characterized by a difference in the AMEX. index during $t_{j+1}, t_{j+2}$ days and subsequent $t_{j+3}, t_{j+4}$ days. 290 days with highest increase in AMEX index and 290 days with highest decrease in this index formed two pattern class data. $50 \%$ of data were used for training and he rest for testing. Ten days history of five oil prices (the number of input features $p=50$ ) were used for classification.

The calculations according to Eq. (3) presented in previous section for 3D and high dimensional new features data have shown that apparent distances increase notably. This evaluation suggests that there is a sense to extract only two or three features. In addition we studied FE accuracy with artificial data formed from original one by means of $k$-NN noise injection [9]. We determined the class number of each vector by classifying this data with MLP with six hidden units.

The experiments with artificial and real data confirmed theoretical conclusion: in situations where a number of new extracted features were four or five, it was impossible to design the classifier capable discriminate the test set vectors in new feature space. Even in 2D new feature space, the best result obtained was: hold out error $\mathrm{PHO}=0.33$ error while training set (resubstitution) error $\mathrm{PR}=0.16$ (see Fig. $1 \mathrm{~b}$ ). In case of three new features, we succeeded to obtain $2 \mathrm{D}$ mappings with $\mathrm{PR}=0.06$. In such cases, however, PHO was close to $50 \%$ error. In order to simplify the feature extraction procedure even more, we formed new 5 times larger pseudo artificial training set. In the regularized 2D data mapping, we did not see clear structure of the data, however, the training set and test set mappings result similar patterns (Fig. 2): the classes can be approximately separated by " $\Gamma$ " shaped DB. The hold our error in 
2D feature space was diminished until 0.26. Thus, proper ignorance of untruthful data structure helped us to improve classification accuracy notably. This analysis confirms earlier conclusion: often in time series prediction, simple forecasting models are more functional [13].

The weights of the neuro-linear classifier often are large and outputs of many test or training vectors are close either to zero or to one (if sigmoid activation function is used). To display the data better in new 2D space or to see differences between classification scores of pattern vectors with identical outputs sometimes it is worth to decrease the weights of each single neuron proportionally, i.e. the output of the neuron $o=f(\beta(w T x+w 0))$. After some experiments, for hidden layer usually we selected $0.2<\beta<0.9$ and for output layer $0.001<\beta<0.1$. Such approach allows distinguish similar outputs and helps the end users to consider economical and political factors not included into the data. In our research, we also used principal components and projection pursuit data mapping to map artificial data mentioned above into 2D space and form two pattern classes. The supervised method, the nonlinear FE, however, was more accurate for the test data discrimination.

In Fig. 3 we plotted the test set instances of the highest increases (circles) and decreases (stars) in the oil prices occurring in a period of $1999-2005$. Instances of mild increase/decrease are marked by "pluses". Our results advocate that in spite of harsh competition in the oil in certain cases it is possible to predict largest increases and decreases in the price. The prediction accuracy may be improved by attracting informal factors into analysis, retraining of the prediction rule for every new day prediction, utilization of multiple classifier systems (see e.g. [14]).

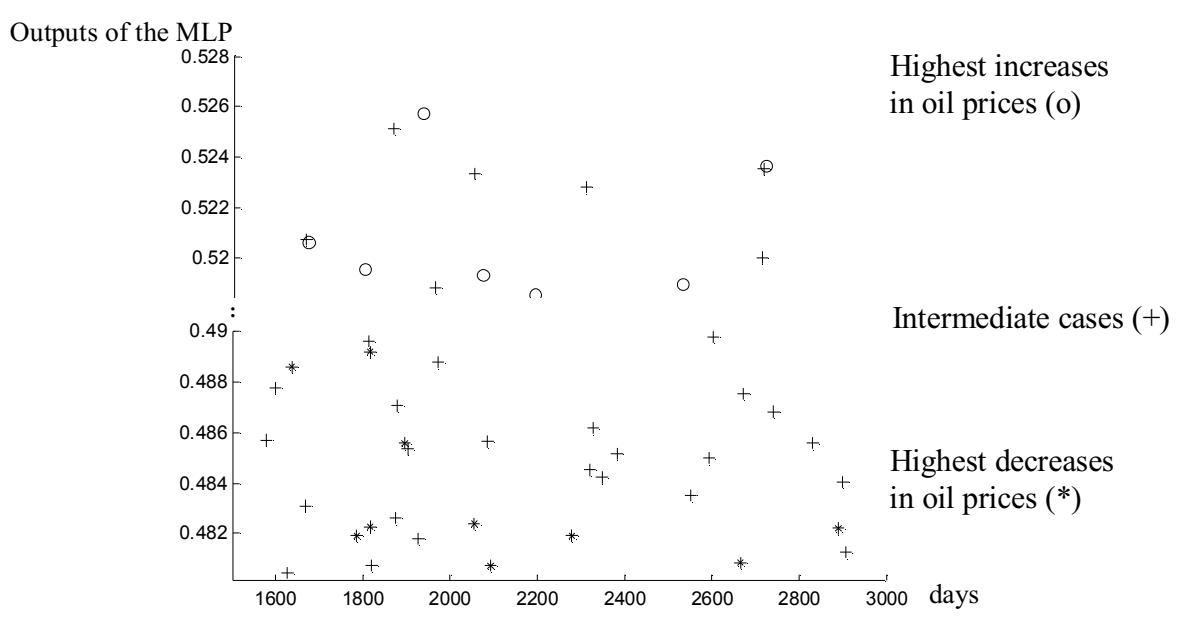

Fig. 3. Visualization of a part of classification results: specially calculated outputs of MLP classifier. Only instances of the highest increases (circles) and decreases (stars) in the oil prices occurring in a period of 1999-2005 are shown. Mild increase/decrease are marked by "pluses". 


\section{Conclusions}

The neuro-linear feature mapping is very simple, however, useful tool for visual inspection of the data. It is a helpful researcher's adviser. To the analyst it is useful in generation of functional hypothesis about the data structure and formation of the pattern classes. The neuro-linear FE where criterion of feature quality depends on dimensionality of the new feature space. We found that an effective sample size actually participating in formation of the new features is decreasing together with an increase in dimensionality of the new feature space. Thus, in finite sample size situations, one needs to reduce a number of new features drastically. Useful tools to simplify data mapping procedure are also training the MLP classifier with artificial training set formed by means of $k$-NN noise injection and utilization of the weight decay term.

In spite of a vast research efforts undertaken in the forecasting task (see e.g. recent review [15]) and a great number of agents competing in forecasting of financial and economical time series, the prophecy may be effective if new unexpected formulation of the problem is undertaken. Informal, interactive, "visual" forecasting procedure could be of great help here. In present paper the data mapping successfully was used for formation of pattern classes in oil price forecasting task. We found that only two extracted features are reliable and useful when training history was composed of six years historical data. This recommendation was productively used also for developing adaptable forecasting algorithms of the sugar, wheat and pork belly prices [16].

Acknowledgments. The author thanks Prof. Sarunas Raudys for indication of useful literature sources and valuable suggestion concerning derivation of Equation (3).

\section{References}

[1] Fama, E.F. Efficient capital markets: A review of theory and empirical work. Journal of Finance, 25: 383-417, 1970.

[2] Fukunaga, K. Introduction to Statistical Pattern Recognition. 2nd ed. Acad. Press, NY, 1990.

[3] Duda, P.E., Hart, R.O. and Stork, D.G. Pattern Classification. 2nd ed. Wiley, NY, 2000.

[4] Raudys, S. Statistical and Neural Classifiers: An integrated approach to design. Springer, NY, 2001.

[5] Raudys, A. and J.A. Long. MLP based linear feature extraction for nonlinearly separable data. Pattern Analysis and Applications, 4(4): 227-234, 2001.

[6] Sammon J.W. An optimal discriminant plane. IEEE Trans Computers, C-19: 826-829, 1970.

[7] Foley D.H. and Sammon J.W.J. An optimal set of discriminant vectors. IEEE Trans. on Computers, C-24: 281-289, 1975.

[8] Koford J.S., Groner G.F. The use of an adaptive threshold element to design a linear optimal pattern classifier. IEEE Transactions on Information Theory, IT-2:42-50, 1966.

[9] Skurichina M., Raudys S., Duin R.P.W. K-nearest neighbors directed noise injection in multilayer perceptron training, IEEE Trans. on Neural Networks, 11: 504-511, 2000

[10] Adamson D.M. Soviet Gas and European-Security, Energy Policy, 13 (1): 13-26, 1985. 
[11] Yousefi A., Wirjanto T.S. The empirical role of the exchange rate on the crude-oil price formation. Energy Economics, 26(5): 783-799, 2004.

[12] Leduc S., Sill K. A quantitative analysis of oil-price shocks, systematic monetary policy, and economic downturns. J. of Monetary Economics, 51(4): 781-808, 2004.

[13] Raudys, A. and Mockus J. Comparison of ARMA and multilayer perceptron based methods for economic time series forecasting. Informatica, 10(2): 231-244, 1999.

[14] Kittler J., Roli F.(eds). Multiple Classifier Systems. Lecture Notes in Computer Science, Springer-Verlag, NY, volumes 1857, 2096, 2364, 2709, 3077 (2000 - 2004).

[15] Wei H., Lai K.K., Nakamori Y., Wang S.Y. Forecasting foreign exchange rates with artificial neural networks: A review. Int. J. on Information Technology and Decision Making, 3(1): 145-165, 2004.

[16] Zliobaite I. and Raudys S. Prediction of commodity prices in rapidly changing environments (Lecture Notes in Computer Science ICAPR'2005 Bath, UK, 2005). 